\title{
A CYCLIC INEQUALITY
}

\author{
by R. A. RANKIN \\ (Received 20th March 1961)
}

\section{Introduction.}

For any positive integer $n$ and positive real variables $x_{1}, x_{2}, \ldots, x_{n}$ write

$$
\begin{aligned}
& S_{n}\left(x_{1}, x_{2}, \ldots, x_{n}\right)=\frac{x_{1}}{x_{2}+x_{3}}+\frac{x_{2}}{x_{3}+x_{4}}+\ldots+\frac{x_{n-1}}{x_{n}+x_{1}}+\frac{x_{n}}{x_{1}+x_{2}} \\
& =\sum_{r=1}^{n} \frac{x_{r}}{x_{r+1}+x_{r+2}}
\end{aligned}
$$

where $x_{r}$ is defined for all integers $r$ by the relations

If

$$
x_{r+n}=x_{r}
$$

$$
\mu(n)=\inf _{\substack{x_{r}>0 \\ 1 \leqq r \leqq n}} S_{n}\left(x_{1}, x_{2}, \ldots, x_{n}\right),
$$

and

it is known that $(1,3,4)$

$$
\lambda(n)=\frac{\mu(n)}{n},
$$

and

$$
\lambda(n)=\frac{1}{2} \quad(n \leqq 6)
$$

$$
\lambda(n)<\frac{1}{2} \quad \text { (even } n \geqq 14 \text {, odd } n \geqq 53 \text { ). }
$$

It is also known that (2) $\lambda(n)$ tends to a limit $\lambda$ as $n \rightarrow \infty$ and that

Further (4),

$$
\lambda=\lim _{n \rightarrow \infty} \lambda(n)=\inf _{n \geqq 1} \lambda(n) \text {. }
$$

$$
\lambda \leqq \lambda(24)<0.49950317 \text {. }
$$

No positive lower bound for $\lambda(n)$, and so for $\lambda$, appears to be known, however, other than the lower bound

$$
\frac{1}{6}(2 \sqrt{ } 2-1)=0 \cdot 3047
$$

stated by me, without proof, in 1957 (2), although the problem of estimating $\lambda(n)$ has aroused considerable interest in the last few years. For this reason $I$ give here an account of the method used to obtain the lower bound (5), which I have since improved, so that I can now prove the

Theorem. $\lambda(n)>0 \cdot 33$ for all $n \geqq 1$.

The lower bound arises as the minimum of the expression

$$
\frac{3}{2} \frac{2^{1-\xi}-\xi}{9-10 \xi}
$$


subject to the condition $0 \leqq \xi \leqq \frac{1}{2}$; a more accurate estimate of this minimum is $0 \cdot 330232$.

As will be realised from its length, the proof of this result is not simple, although the ideas involved are fairly elementary. The details are abbreviated as much as possible.

\section{Some Lemmas.}

We shall make considerable use of the functions $f$ and $g$ which are defined on the non-negative numbers as follows:

$$
\begin{array}{llll}
f(x)=x & \left(0 \leqq x \leqq \frac{1}{2}\right), & f(x)=\sqrt{ }(2 x)-\frac{1}{2} & \left(x \geqq \frac{1}{2}\right), \\
g(x)=x & (0 \leqq x \leqq 1), & g(x)=1+\log x & (x \geqq 1) .
\end{array}
$$

Lemma 1. $f(x)$ and $g(x)$ are convex functions of $\log x$ for $x>0$; i.e., for any positive integer $m$ and positive $x_{1}, x_{2}, \ldots, x_{m}$

$$
f\left(x_{1}\right)+f\left(x_{2}\right)+\ldots+f\left(x_{m}\right) \geqq m f\left\{\left(x_{1} x_{2} \ldots x_{m}\right)^{1 / m}\right\},
$$

and $g$ satisfies a similar inequality. Further

$$
x \geqq f(x) \geqq \sqrt{ }(2 x)-\frac{1}{2} \text { for } x \geqq 0 .
$$

Proof. Both $f(x)$ and $g(x)$ are continuous and have continuous derivatives for $x \geqq 0$. They possess second derivatives $f^{\prime \prime}(x)$ and $g^{\prime \prime}(x)$ except for $x=\frac{1}{2}$ and $x=1$, respectively, and

$$
x f^{\prime \prime}(x)+f^{\prime}(x) \geqq 0, \quad x g^{\prime \prime}(x)+g^{\prime}(x) \geqq 0,
$$

except at these points, from which the convexity properties follow. The last inequality for $f$ is easily verified.

In the following four lemmas we are concerned with finding lower bounds for partial sums of (1); we write, for any positive integer $L$,

$$
\phi_{L}=\phi_{L}\left(x_{0}, x_{1}, \ldots, x_{L+1}\right)=\sum_{r=0}^{L-1} \frac{x_{r}}{x_{r+1}+x_{r+2}},
$$

where $x_{0}, x_{1}, \ldots, x_{L+1}$ are any $L+2$ positive numbers, subject only to the restrictions imposed in the lemmas.

Lemma 2. If $L \geqq 1$ and $x_{1} \geqq x_{2} \geqq \ldots \geqq x_{L+1}$, then $\phi_{L} \geqq \frac{1}{2} L\left(x_{0} / x_{L}\right)^{1 / L}$.

Proof. By the inequality of the arithmetic and geometric means,

$$
\begin{aligned}
\phi_{L} & \geqq L\left\{\frac{x_{0} x_{1} \ldots x_{L-1}}{\left(x_{1}+x_{2}\right)\left(x_{2}+x_{3}\right) \ldots\left(x_{L}+x_{L+1}\right)}\right\}^{1 / L} \\
& =L\left\{\frac{x_{0}}{x_{L}+x_{L+1}} \cdot \frac{1}{1+\frac{x_{2}}{x_{1}}} \ldots \frac{1}{1+\frac{x_{L}}{x_{L-1}}}\right\}^{1 / L} \\
& \geqq L\left\{\frac{x_{0}}{2 x_{L}} \cdot \frac{1}{2^{L-1}}\right\}^{1 / L} \cdots=\frac{1}{2} L\left(x_{0} / x_{L}\right)^{1 / L} .
\end{aligned}
$$


Lemma 3. If $L=2$ and $x_{1} \leqq x_{2}, x_{3} \leqq x_{2}$, then $\phi_{2} \geqq f\left(x_{0} / x_{2}\right)$.

Proof. For $x \geqq 0$ and $0 \leqq u \leqq 1$, write

so that

$$
h(x, u)=\frac{x}{1+u}+\frac{1}{2} u
$$

We prove that

$$
\begin{aligned}
\phi_{2} & =\frac{x_{0}}{x_{1}+x_{2}}+\frac{x_{1}}{x_{2}+x_{3}} \geqq \frac{x_{0}}{x_{1}+x_{2}}+\frac{x_{1}}{2 x_{2}} \\
& =h\left(\frac{x_{0}}{x_{2}}, \frac{x_{1}}{x_{2}}\right) \ldots \ldots \ldots \ldots \ldots \ldots \ldots \ldots \ldots \ldots \ldots \ldots \ldots \ldots \ldots
\end{aligned}
$$

$$
h(x, u) \geqq f(x) \quad(x \geqq 0,0 \leqq u \leqq 1) .
$$

For $0 \leqq x \leqq \frac{1}{2}$ this follows since

$$
h(x, u)-f(x)=\frac{u(1+u-2 x)}{2(1+u)} .
$$

If $x>\frac{1}{2}$ it is easily verified that, for fixed $x, h(x, u)$ has a minimum where $u=\sqrt{ }(2 x)-1$, so that

$$
h(x, u) \geqq h(x, \sqrt{ }(2 x)-1)=f(x) .
$$

The result follows from (8) and (9).

Lemma 4. If $L=3$ and $x_{1} \leqq x_{2} \leqq x_{3}, x_{4} \leqq x_{3}$, then $\phi_{3} \geqq g\left(x_{0} / x_{3}\right)$.

Proof. We have, by (6) and (7) and (9),

$$
\begin{aligned}
\phi_{3} & \geqq \frac{x_{0}}{x_{1}+x_{3}}+\frac{x_{1}}{x_{2}+x_{3}}+\frac{x_{2}}{2 x_{3}} \\
& =\frac{x_{0}}{x_{1}+x_{3}}+h\left(\frac{x_{1}}{x_{3}}, \frac{x_{2}}{x_{3}}\right) \\
& \geqq \frac{x_{0}}{x_{1}+x_{3}}+f\left(\frac{x_{1}}{x_{3}}\right) \\
& =H\left(\frac{x_{0}}{x_{3}}, \frac{x_{1}}{x_{3}}\right), \ldots . . . . . . . .
\end{aligned}
$$

where

$$
H(x, u)=\frac{x}{1+u}+f(u)
$$

and we suppose that $x \geqq 0,0 \leqq u \leqq 1$. We prove that

$$
\psi(x, u)=\frac{x}{1+u}+f(u)-g(x) \geqq 0
$$

and may clearly suppose that $u>0$. Since

$$
\psi_{x}(x, u)=\frac{1}{1+u}-g^{\prime}(x)
$$


there is, for fixed $u$, a minimum where $x=1+u$, and so

$$
\psi(x, u) \geqq \psi(1+u, u)=f(u)-\log (1+u) .
$$

This last expression has a non-negative derivative for $u \geqq 0$, so that

$$
\psi(x, u) \geqq f(0)-\log 1=0 .
$$

This proves (11), and the lemma follows, by (10).

Lemma 5. If $L \geqq 4$ and $x_{1} \leqq x_{2} \leqq \ldots \leqq x_{L}, x_{L+1} \leqq x_{L}$, then

$$
\phi_{L} \geqq \frac{1}{2}(L-1) f\left\{\left(x_{0} / x_{L}\right)^{2 /(L-1)}\right\} .
$$

Proof. We have, by (7), (9) and Lemma 1,

$$
\begin{aligned}
\phi_{L} & \geqq \frac{x_{0}}{x_{1}+x_{3}}+\frac{x_{1}}{2 x_{3}}+0+\frac{x_{3}}{2 x_{5}}+\frac{x_{4}}{2 x_{6}}+\ldots+\frac{x_{L-2}}{2 x_{L}}+\frac{x_{L-1}}{2 x_{L}} \\
& \geqq h\left(x_{0} / x_{3}, x_{1} / x_{3}\right)+\frac{1}{2}(L-3)\left\{\frac{x_{3} x_{4}}{x_{L}^{2}}\right\}^{1 /(L-3)} \\
& \geqq f\left(x_{0} / x_{3}\right)+\frac{1}{2}(L-3)\left(x_{3} / x_{L}\right)^{2 /(L-3)} \\
& \geqq f\left(x_{0} / x_{3}\right)+\frac{1}{2}(L-3) f\left\{\left(x_{3} / x_{L}\right)^{2 /(L-3)}\right\} \\
& \geqq \frac{1}{2}(L-1) f\left\{\left(x_{0} / x_{L}\right)^{2 /(L-1)}\right\} .
\end{aligned}
$$

The functions $f$ and $g$, whose values appear as lower bounds in Lemmas 3 , 4 and 5 , can be replaced by functions taking larger values over certain parts of their domains; thus, for $1 \leqq x \leqq 9 / 4,1+\log x$ can be replaced by $2 \sqrt{ } x-1$. These improvements, however, produce no corresponding improvements in the main lemma, which is Lemma 7, and so are not given.

Lemma 6. Let $a, b, c, d, A, B$ and $C$ be non-negative constants with $A+B+C$ $=1$. Then, if $x, y$ and $z$ are any non-negative numbers such that

we have

$$
x^{A} y^{B} z^{c}=d
$$

$$
a A x+b B y+c C z \geqq d a^{A} b^{B} c^{c},
$$

where $0^{\circ}$ is always to be interpreted as 1 .

The proof is straightforward and is omitted.

Lemma 7. Let

$$
F(x, y, z, t)=p x+q^{\prime}\left(y \sqrt{ } 2-\frac{1}{2}\right)+r\left(z \sqrt{ } 2-\frac{1}{2}\right)+2 s g(t),
$$

where $x, y, z$ and $t$ are positive numbers satisfying

$$
x^{p} y^{q^{\prime}} z^{r} t^{s}=1
$$

and $p, q, q^{\prime}, r$ and $s$ are non-negative numbers such that

Then

$$
q^{\prime} \geqq \frac{3}{4} q, \quad p+q+r+3 s=n>0 .
$$

$$
F(x, y, z, t)>0.66 n \text {. }
$$


Proof. We put $m=p+q^{\prime}+r$. If $m=0$, then $s=\frac{1}{3} n, t=1$ and

$$
F(x, y, z, t)=\frac{2}{3} n g(1)=\frac{2}{3} n .
$$

We may therefore suppose that $m>0$, and put

so that

$$
\text { , }=m u, \quad q^{\prime}=m v, \quad s=m w,
$$

$$
m\left(1+\frac{1}{3} v+3 w\right) \geqq n .
$$

By Lemma 6, with $a=m, b=c=m \sqrt{ } 2, A=u, B=v$,

$$
F(x, y, z, t) \geqq m\left\{t^{-w} 2^{\frac{1}{(1-u)}}-\frac{1}{2}(1-u)+2 w g(t)\right\}=F_{1},
$$

say. We find the minimum of $F_{1}$ subject to (12) and the inequalities

We have

$$
t \geqq 0, \quad 0 \leqq u \leqq 1-v, \quad w \geqq 0 .
$$

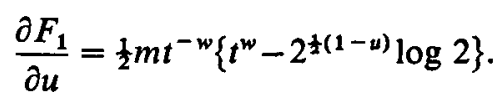

It is convenient to write

$$
\alpha=\log 2=0.69315, \quad \beta=-\log \log 2=0.36651,
$$

all decimals being correct to the number of places given. There are three cases to be considered: (i) $\alpha 2^{\frac{1}{2} v} \leqq t^{w} \leqq \alpha 2^{\frac{1}{t}}$, (ii) $t^{w} \leqq \alpha 2^{\frac{1}{t} v}$, and (iii) $t^{w} \geqq \alpha 2^{\frac{1}{2}}$.

(i) In this case $t<1$, since $\alpha 2^{\frac{1}{2}}=0.98026<1$, and the inequalities for $t^{w}$ may be expressed in the form

$$
\frac{\alpha-2 \beta}{2 \log t} \leqq w \leqq \frac{v \alpha-2 \beta}{2 \log t} .
$$

It follows from (13) that $F_{1}$ has a minimum where $\alpha 2^{\frac{1}{(1-u)}}=t^{w}$, so that

$$
F_{1} \geqq F_{2}=m \alpha^{-1}\{1-\beta+w(2 \alpha t-\log t)\}>0 .
$$

We suppose first that $0<t \leqq \frac{1}{2}$; since $2 \alpha t-\log t$ decreases with $t$,

since $9(1-\beta)-8 \alpha>0$.

$$
\begin{aligned}
F_{2} & \geqq m \alpha^{-1}(1-\beta+2 \alpha w) \\
& \geqq \frac{n}{\alpha} \frac{1-\beta+2 \alpha w}{\frac{4}{3}+3 w} \geqq \frac{2}{3} n,
\end{aligned}
$$

We may therefore suppose that $\frac{1}{2}<t<1$. Then

and $\partial F_{3} / \partial w<0$, since

$$
F_{2} \geqq F_{3}=n \frac{1-\beta+w(2 \alpha t-\log t)}{\alpha\left(1+\frac{1}{3} v+3 w\right)},
$$

$$
\left(1+\frac{1}{3} v\right)(2 \alpha t-\log t)-3(1-\beta)<2 \alpha\left(1+\frac{1}{3} v\right)-3(1-\beta) \leqq \frac{8}{3} \alpha-3(1-\beta)<0 .
$$

Hence, by (14),

$$
F_{3} \geqq F_{4}=\frac{n}{\alpha} \frac{A-B v}{C-D v}
$$


where

$$
\begin{array}{ll}
A=2 \alpha \beta t-\log t, \quad \beta=\frac{1}{2} \alpha(2 \alpha t-\log t), \\
C=3 \beta-\log t, \quad D=\frac{3}{2} \alpha+\frac{1}{3} \log t .
\end{array}
$$

We show that $F_{4} \geqq \frac{2}{3} n$; this will follow if we prove that

Now

$$
3 A-2 \alpha C \geqq v(3 B-2 \alpha D) \text {. }
$$

$$
3 B-2 \alpha D=-3 \alpha\left\{\alpha(1-t)+\frac{13}{18} \log t\right\},
$$

which decreases for $\frac{1}{2}<t<1$, since $18 \alpha<13$, and so exceeds zero. Hence we can take $v=1$ in (15) and must prove that

i.e. that

$$
3(A-B) \geqq 2 \alpha(C-D),
$$

Now

$$
\psi(t)=-(18-25 \alpha) \log t-18 \alpha(2 \beta-\alpha)(1-t)>0 \text {. }
$$

$$
\begin{aligned}
\psi^{\prime}(t) & =18 \alpha(2 \beta-\alpha)-(18-25 \alpha) t^{-1} \\
& =0.49755-0.67132 t^{-1}<0
\end{aligned}
$$

so that $\psi(t) \geqq \psi(1)=0$, as required. Thus we have shown that, if case (i) holds, then $F_{1} \geqq \frac{2}{3} n$.

(ii) In this case $0<t<1$ and

$$
w \geqq \frac{v \alpha-2 \beta}{2 \log t}>0 .
$$

Then $\partial F_{1} / \partial u \leqq 0$, by (13), so that, since $u \leqq 1-v$,

$$
F_{1} \geqq n F_{5}=n \frac{t^{-w} 2^{\frac{1}{2} v}-\frac{1}{2} v+2 w t}{1+\frac{1}{3} v+3 w} \text {. }
$$

The numerator is positive since $2^{\frac{1}{2} v}>\frac{1}{2} v$. Regarded as a function of $t, F_{5}$ has a minimum at

We put

$$
t=2^{-\xi}, \text { where } \xi=\frac{1-\frac{1}{2} v}{1+w} .
$$

so that

$$
\eta=\frac{1}{1+w}
$$

$$
v=2\left(1-\frac{\xi}{\eta}\right), \quad w=\frac{1}{\eta}-1 .
$$

Now $0 \leqq v \leqq 1$ and so, by (16),

and

$$
0<\eta \leqq 2 \xi \leqq 2 \eta \leqq 2
$$

We have

$$
\xi \leqq 1-\frac{\beta}{\alpha}=0 \cdot 47123 \text {. }
$$

$$
F_{5}=F_{5}(\xi, \eta)=3 \frac{2^{1-\xi}+\xi-\eta}{9-2 \xi-4 \eta}
$$


and

$$
(9-2 \xi-4 \eta)^{2} \frac{\partial F_{5}}{\partial \eta}=3\left(2^{3-\xi}+6 \xi-9\right)<0
$$

for $2^{3-\xi}+6 \xi-9<0$ for $\xi<\frac{1}{2}$. Thus $F$ decreases with $\eta$ and so, since $\eta \leqq 2 \xi<1$,

$$
F_{5} \geqq F_{5}(\xi, 2 \xi)=F_{6}(\xi)=3 \frac{2^{1-\xi}-\xi}{9-10 \xi} .
$$

It therefore suffices to show that

i.e. that

$$
F_{6}(\xi)>0.66 \text { for } 0 \leqq \xi \leqq \frac{1}{2} \text {, }
$$

$$
F_{7}(\xi)=2^{1-\xi}+1 \cdot 2 \xi-1 \cdot 98>0 .
$$

This holds since $F_{7}(\xi)$ has a minimum where $2^{\xi}=\frac{5}{3} \log 2$, so that

$$
F_{7}(\xi) \geqq \frac{6}{5} \frac{\log \left(\frac{5}{3} e \log 2\right)}{\log 2}-1.98=0.00107>0 .
$$

(iii) In this case we can again show that $F_{1} \geqq \frac{2}{3} n$. We have $\partial F_{1} / \partial u \geqq 0$, so that and

$$
F_{1} \geqq F_{8}=m\left\{t^{-w} 2^{\frac{1}{2}}-\frac{1}{2}+2 w g(t)\right\}
$$

$$
\frac{\partial F_{8}}{\partial t}=m w\left\{2 g^{\prime}(t)-2^{\frac{1}{2}} t^{-w-1}\right\} .
$$

If $t \geqq 1, g^{\prime}(t)=t^{-1}$ so that $\partial F_{8} / \partial t>0$ and

since $9\left(2^{\frac{1}{2}}-\frac{1}{2}\right)>8$.

$$
\begin{aligned}
F_{8} & \geqq m\left(2^{\frac{1}{2}}-\frac{1}{2}+2 w\right) \\
& \geqq n \frac{2^{\frac{1}{2}}-\frac{1}{2}+2 w}{\frac{4}{3}+3 w} \geqq \frac{2}{3} n,
\end{aligned}
$$

We may therefore suppose that $t<1$ and we then have

$$
F_{1} \geqq n \frac{t^{-w} 2^{\frac{1}{2}}-\frac{1}{2}+2 w t}{\frac{4}{3}+3 w} \text {. }
$$

By (17), the right-hand side is the same as $n F_{5}$ with $v=1$ and so has a minimum for $t=2^{-\xi}$, where $\xi=1 /\{2(1+w)\}$. Now $v=1$ gives $\eta=2 \xi$, so that, by (18),

$$
F_{1} \geqq n F_{6}(\xi) \text {. }
$$

The conditions on $\xi$ are different in this case, however. They are

$$
1-\frac{\beta}{\alpha} \leqq \xi \leqq \frac{1}{2} \text {. }
$$

It is easily verified that $9 \cdot 2^{1-\xi}>18-11 \xi$ in this range, so that $F_{6}(\xi)>\frac{2}{3}$. This completes the proof of the lemma.

3. Proof of the Theorem. Let $x_{1}, x_{2}, \ldots, x_{n}$ be any $n$ positive numbers. We prove that

$$
\text { E.M.S. }- \text { L }
$$

$$
S_{n}\left(x_{1}, x_{2}, \ldots, x_{n}\right)>0 \cdot 33 n \text {. }
$$


We may suppose that the $n$ numbers are not all equal, since otherwise $S_{n}=\frac{1}{2} n$. We divide them into disjoint subsets of numbers with consecutive suffixes by the following method, which we first describe in general terms.

Because of our convention (2), we may regard the $n$ numbers as being arranged in cyclic order, for example, on the circumference of a circle. This circumference we divide into subsets by making a cut before every minimum and after every maximum. This yields subsets of two types, (i) increasing subsets running from a minimum to a maximum, and (ii) decreasing subsets running from just after a maximum to just before a minimum.

More precisely, if $L \leqq n$, a subset $x_{q+1}, x_{q+2}, \ldots, x_{q+L}$ is called an increasing subset of length $L$, if $L \geqq 2$ and

$$
x_{q}>x_{q+1}, x_{q+1} \leqq x_{q+2} \leqq \ldots \leqq x_{q+L}, x_{q+L}>x_{q+L+1} .
$$

It is called a decreasing subset of length $L$, if $L \geqq 1$ and

$$
x_{q}>x_{q+1} \geqq x_{q+2} \geqq \ldots \geqq x_{q+L}>x_{q+L+1} .
$$

In either case $x_{q} / x_{q+L}$ is called the ratio of the subset.

In this way the set of $n$ numbers $x_{1}, x_{2}, \ldots, x_{n}$ is divided into a number of disjoint subsets, the product of whose ratios is 1 . Two increasing subsets may follow one after the other, but two or more consecutive decreasing subsets can always be combined into a single decreasing subset, and we shall suppose that this is done.

The sum $S\left(x_{1}, x_{2}, \ldots, x_{n}\right)$ also splits up into a number of subset sums, one from each subset; for example, the contribution to $S\left(x_{1}, x_{2}, \ldots, x_{n}\right)$ from the subset $x_{q+1}, x_{q+2}, \ldots, x_{q+L}$ is

$$
\sum_{r=0}^{L-1} \frac{x_{q+r}}{x_{q+1+r}+x_{q+2+r}}
$$

and is a sum of the form (6). Lemmas 2 to 5 are applicable to these subset sums. In fact Lemma 2 applies to decreasing subsets of any length $L \geqq 1$, while Lemmas 3,4 and 5 apply to increasing subsets of lengths 2,3 and $L \geqq 4$, respectively. We can split $S=S\left(x_{1}, x_{2}, \ldots, x_{n}\right)$ into four corresponding parts, namely

$$
S=D+I_{2}+I_{3}+I_{4},
$$

where, for example, $I_{4}$ denotes the sum of all increasing subset sums of length $L \geqq 4$.

If $p$ is the total length of all decreasing subsets, Lemma 2 combined with the inequality of the arithmetic means shows that

$$
D \geqq \frac{1}{2} p x,
$$

where $x^{p}$ is the product of all the ratios associated with these subsets. Similarly, Lemmas 1 and 3 show that

$$
I_{2} \geqq \frac{1}{2} r f\left(z^{2}\right),
$$




\section{A CÝCLIC INEQUALITY}

where $r$ is the total length of all increasing subsets of length 2 and $z^{r}$ is the product of their ratios. Also, Lemmas 1 and 4 show that

$$
I_{3} \geqq \operatorname{sg}(t) \text {, }
$$

where $3 s$ is the total length of all increasing subsets of length 3 and $t^{s}$ is the product of their ratios. Finally, if

$$
q^{\prime}=\Sigma(L-1), \quad q=\Sigma L,
$$

the summations being over all lengths $L \geqq 4$ of increasing subsets, Lemmas 1 and 5 show that

$$
I_{4} \geqq \frac{1}{2} q^{\prime} f\left(y^{2}\right),
$$

where $y^{q^{\prime}}$ is the product of their ratios. By the last part of Lemma 1 we have

$$
2 S \geqq p x+q^{\prime}\left(y \sqrt{ } 2-\frac{1}{2}\right)+r\left(z \sqrt{ } 2-\frac{1}{2}\right)+2 s g(t)
$$

and, since $L-1 \geqq \frac{3}{4} L$ for $L \geqq 4$,

$$
q^{\prime} \geqq \frac{3}{4} q \text { and } p+q+r+3 s=n .
$$

Also, since the product of all the ratios is 1 ,

$$
x^{p} y^{q^{\prime}} z^{r} t^{s}=1
$$

The Theorem now follows from Lemma 7 .

\section{REFERENCES}

(1) L. J. MoRdelL, On the inequality $\sum_{r=1}^{n} x_{r} /\left(x_{r+1}+x_{r+2}\right) \geqq \frac{1}{2} n$ and some others, Abh. Math. Sem. Univ. Hamburg, 22 (1958), 229-240.

(2) R. A. Rankin, An inequality, Math. Gaz. 42 (1958), 39-40.

(3) A. Zulauf, Note on a conjecture of L. J. Mordell, Abh. Math. Sem. Univ. Hamburg, 22 (1958), 241.

(4) A. Zulauf, On a conjecture of L. J. Mordell II, Math. Gaz., 43 (1959), 182-184.

THE UNIVERSITY

GLASGOW 